\title{
Indicios de fibrogénesis en hígado esteatósico no alcohólico de pacientes con componentes del síndrome metabólico
}

\section{Evidence of fibrogenesis in non-alcoholic steatotic liver of patients with components of the metabolic syndrome}

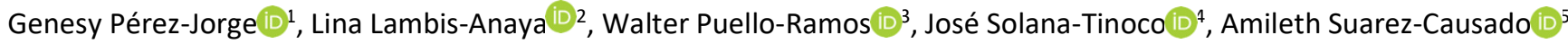 \\ 1. Universidade Estadual de Campinas. Campinas, Brasil. Correo: g211546@dac.unicamp.br - https://orcid.org/0000-0002-2138-7393 \\ 2. Universidad de Cartagena. Cartagena, Colombia. Correo: llambisa1@unicartagena.edu.co - https://orcid.org/0000-0002-5894-2530 \\ 3. Universidad de Cartagena. Cartagena, Colombia. Correo: walterpuello@yahoo.com - https://orcid.org/0000-0002-0156-5762 \\ 4. Universidad de Cartagena. Cartagena, Colombia. Correo: jsolanat@unicartagena.edu.co - https://orcid.org/0000-0002-3748-2678 \\ 5. Universidad de Cartagena. Cartagena, Colombia. Correo: asuarezc1@unicartagena.edu.co - https://orcid.org/0000-0003-2807-0679
}

Tipología: Artículo de investigación científica y tecnológica

Para citar este artículo: Pérez-Jorge G, Lambis-Anaya L, Puello-Ramos W, Solana-Tinoco J, Suarez-Causado A. Indicios de fibrogénesis en hígado esteatósico no alcohólico de pacientes con componentes del síndrome metabólico. Duazary. 2021 abril; 18(2): 141-152. Doi: https://doi.org/10.21676/2389783X.4077

Recibido en agosto 22 de 2020

Aceptado en febrero 15 de 2021

Publicado en línea en mayo 25 de 2021

\section{Palabras \\ clave: \\ esteatosis \\ hepática no \\ alcohólica; \\ fibrosis; \\ síndrome \\ metabólico; \\ obesidad; \\ expresión \\ génica.}

\section{RESUMEN}

La esteatosis no alcohólica es asociada con pronóstico benigno. Sin embargo, puede progresar a fibrosis, aunque no se comprende completamente el comportamiento de los genes que contribuyen a esta progresión. El objetivo de esta investigación fue analizar los indicios tempranos de fibrogénesis en la esteatosis no alcohólica en pacientes obesos frente a no obesos. Se realizó un estudio descriptivo prospectivo que incluyó a pacientes con esteatosis no alcohólica. Estos fueron divididos en dos grupos: obesos y no obesos. Se obtuvo información sociodemográfica, antropométrica y de padecimientos de componentes del síndrome metabólico. Se determinaron parámetros bioquímicos y niveles de expresión de los genes TNF- $\alpha$, NF-k $\beta$, CYP2E1, TGF- $\beta$, OPN, COL4 $\alpha 5$ y HGF por RT-PCR. Los resultados muestran diferencias significativas en los niveles de expresión del gen CYP2E1, siendo mayor en pacientes obesos que padecían síndrome metabólico completo. Además, en pacientes no obesos sin componentes del síndrome metabólico se activa TGF- $\beta$ como participante de vías fibrogénicas, y en pacientes obesos con síndrome metabólico completo el nivel de expresión significativo de CYP2E1 podría indicar una señal de alarma por un posible progreso de la enfermedad. Debe prestarse especial atención y seguimiento de la esteatosis en pacientes obesos.

\section{ABSTRACT}

Keywords: Non-alcoholic hepatic steatosis; Fibrosis; Metabolic syndrome; Obesity; Gene expression.
Non-alcoholic steatosis is generally associated with benign prognosis, however it can progress to fibrosis; the behavior of genes that contribute to their progression to fibrosis are not fully understood yet. The objective of this study was to analize the first signs of fibrogenesis in non-alcoholic steatosis in obese versus non-obese patients. A prospective descriptive study included patients with non-alcoholic steatosis was performed. The patients were divided in two groups, obese and non-obese. Sociodemographic, anthropometric and the suffering of the components of metabolic syndrome information were obtained. Biochemical parameters and expression leves of theTNF- $\alpha$, NF-k $\beta$, CYP2E1, TGF- $\beta$, OPN, COL4 $\alpha 5$ and HGF genes were determined by RT-PCR. Our results showed significant differences in the levels of expression of the CYP2E1 gene, the level of expression being higher in obese patients suffering from complete metabolic syndrome. Furthermore, in non-obese patients with steatosis and without components of the metabolic syndrome, TGF- $\beta$ is activated as a participant in fibrogen pathways and in obese patients with complete metabolic syndrome, the significant expression level of CYP2E1 could indicate an alarm signal 
for possible progress of the illness. Special attention and monitoring of steatosis should be paid in obese patients.

\section{INTRODUCCIÓN}

La enfermedad de hígado graso no alcohólico (NAFLD por sus siglas en inglés: Non-Alcoholic Fatty Liver Disease) es una de las principales causas de morbilidad por enfermedades hepáticas en todo el mundo ${ }^{1}$. NAFLD abarca un espectro de enfermedades que podrían ir desde la esteatosis, la esteatohepatitis y la fibrosis hasta, en última instancia, la cirrosis, enfermedad que aumenta el riesgo de desarrollar carcinoma hepatocelular en algunos individuos ${ }^{2}$. El progreso de NAFLD a fibrosis solo se presenta en una minoría de casos y se debe al resultado de una combinación de factores genéticos, ambientales y metabólicos ${ }^{3}$. Se consideraba que el progreso a fibrosis se daba a partir de la esteatohepatitis; sin embargo, en los últimos años estudios han sugerido que la progresión a fibrosis también ocurre en individuos con esteatosis ${ }^{3-5}$, lo que implicaría que puede no ser una condición totalmente benigna, no progresiva y autolimitada ${ }^{6}$.

Estudios previos que analizaron factores genéticos y metabólicos involucrados en las vías de señalización que llevan al desarrollo y/o progresión de NAFLD han reportado la sobreexpresión de genes como el factor de crecimiento transformante beta (TGF- $\beta$ ), el factor de necrosis tumoral alfa (TNF- $\alpha$ ), el citocromo P450 2E1 (CYP2E1), el factor de crecimiento fibroblástico, entre otros ${ }^{7-9}$. Sin embargo, los mecanismos de señalización génica siguen sin estar claros debido a la complejidad de la patogénesis de esta enfermedad, sumada a las limitaciones que trae consigo el uso de modelos celulares o animales y los escasos estudios realizados en individuos no obesos con esteatosis.

Por lo tanto, es crucial conocer y analizar los genes involucrados en las vías de señalización que conducen a fibrosis en individuos obesos y no obesos con esteatosis y la contribución del síndrome metabólico (SM) en la progresión de esta enfermedad. De esta manera será posible desarrollar estrategias de diagnóstico útiles en etapa inicial de la enfermedad y contribuir a la intervención precoz en los individuos con mayor riesgo de progresión a fibrosis. Este estudio se planteó como objetivo analizar la expresión de genes involucrados en los indicios tempranos de fibrogénesis en la esteatosis no alcohólica en pacientes obesos frente a no obesos y la contribución del SM.

\section{MATERIALES Y MÉTODOS}

\section{Tipo de investigación y participantes}

Estudio descriptivo prospectivo que incluyó pacientes con esteatosis diagnosticada por criterios clínicos y análisis ecográfico, quienes acudieron a la Unidad de Hígado y Vías Biliares del Hospital Universitario del Caribe de Cartagena, durante el periodo de octubre de 2015 a julio de 2017.

Fueron excluidos individuos con consumo de alcohol superior a $10 \mathrm{~g} /$ día para las mujeres y $20 \mathrm{~g} /$ día para los hombres $y / o$ con antecedentes de consumo excesivo de alcohol, evidencia clínica sugestiva de enfermedad hepática inducida por fármacos, uso de fármacos hepatotóxicos (amiodarona, glucocorticoides, tamoxifeno, metrotexate e isoniazida) dentro de los últimos seis meses previos al estudio, antecedentes personales de hepatitis viral, hemocromatosis, hepatitis autoinmune, enfermedad de Wilson, esteatopatitis y cirrosis.

A los pacientes, previo consentimiento informado, se les revisó la historia clínica y se les realizaron pruebas bioquímicas a partir de una muestra de sangre. También se tomó una muestra de biopsia hepática de cada paciente para los estudios moleculares, obtenida por el equipo de hepatólogos de la Unidad de Hígado y Vías Biliares del Hospital Universitario del Caribe de Cartagena.

\section{Procedimiento}

La detección de esteatosis se realizó por ultrasonografía abdominal total con ecógrafo SonoAce 8000 Live (Medison Co. Ltd.) en los pacientes del estudio para determinar el diagnóstico 
y el grado de esteatosis. Se recurrió a la ecografía en este estudio porque es una técnica ampliamente utilizada, segura y no invasiva. Además, estudios que comparan la ecografía y la biopsia hepática muestran que la primera posee una sensibilidad del $90 \%$ y una especificidad del $80 \%{ }^{10}$.

Todas las ultrasonografías fueron realizadas y analizadas por un único medico radiólogo del Hospital Universitario del Caribe con amplia experiencia y en un mismo equipo. Se consideró esteatosis cuando el hígado presentaba $>5 \%$ de infiltración grasa. Además, la esteatosis se clasificó cualitativamente en tres grados conforme a la clasificación usada por Mustapic et $a l^{11}$, esto es, grado 1 o esteatosis leve: hígado ligeramente más brillante en comparación con la corteza renal, visualización clara del diafragma y venas hepáticas con contornos agudos; grado 2 o esteatosis moderada: hígado más brillante con haz atenuado en las partes más profundas, diafragma y venas hepáticas aún visibles pero con contornos romos; grado 3 o esteatosis severa: hígado muy brillante y diafragma o venas hepáticas no visibles.

Las características sociodemográficas y antropométricas y los antecedentes de padecimiento de componentes del SM de cada paciente se tomaron a partir de la historia clínica y de entrevista con los pacientes o familiares de estos. También se consultaron por esta vía los valores de tiempo de protrombina (TP) y tiempo parcial de tromboplastina (TPT). Según el índice de masa corporal (IMC), los pacientes fueron divididos en dos grupos: obesos (IMC $>30 \mathrm{~kg} / \mathrm{m}^{2}$ ) y no obesos $\left(\mathrm{IMC}<30 \mathrm{~kg} / \mathrm{m}^{2}\right)$. Dentro de los dos grupos los pacientes se consideraron con síndrome metabólico completo (SMC) si tenían diabetes mellitus (DM) y a su vez dos de las siguientes enfermedades: hipertensión arterial (HTA), obesidad o dislipidemia; no obstante, si tenían por lo menos uno de los padecimientos mencionados eran considerados con síndrome metabólico incompleto (SMI).

\section{Técnicas}

Análisis bioquímico sanguíneo: para el análisis bioquímico se recolectó una muestra de sangre periférica tras 12 horas de ayuno, a partir de la cual se determinaron concentraciones plasmáticas de los siguientes analitos, teniendo en cuenta los valores de referencia: glucosa $(70-105 \mathrm{mg} / \mathrm{dL})$, colesterol total (hasta $200 \mathrm{mg} / \mathrm{dL}$ ), triglicéridos (hasta 150 $\mathrm{mg} / \mathrm{dL}$ ), bilirrubina (total: hasta $1,1 \mathrm{mg} / \mathrm{dL}$; directa: hasta $0,25 \mathrm{mg} / \mathrm{dL}$; indirecta: hasta $0,76 \mathrm{mg} / \mathrm{dL}$ ), albúmina (3,4-4,8 g/dL) y creatinina (hombres: 0,6$1,1 \mathrm{mg} / \mathrm{dL}$; mujeres: 0,5-1,0 mg/dL). Como se describió ${ }^{12}$, también se determinó la actividad enzimática de aspartato aminotransferasa (AST: hasta $40 \mathrm{U} / \mathrm{L}$ ), alanino aminotransferasa (ALT: hasta $41 \mathrm{U} / \mathrm{L}$ ) y fosfatasa alcalina (ALP: hombres: <270 U/L; mujeres <240 U/L) por los métodos de la IFCC (International Federation of Clinical Chemistry), empleando los kits de Biosystems ${ }^{\circledR}$ (Barcelona, España) correspondientes en cada caso. Todas las mediciones se realizaron siguiendo las recomendaciones del fabricante.

Análisis de la expresión génica mediante reacción en cadena de la polimerasa con transcriptasa inversa (RT-PCR): las muestras de tejido hepático fueron tomadas por un hepatólogo del Hospital Universitario del Caribe. A partir de ellas se realizó la extracción de ARN total empleando el reactivo Qiazol $^{\circledR}$ (Qiagen, California, USA), siguiendo las instrucciones del fabricante. La concentración y la calidad del ARN se verificaron mediante espectrofotometría (NanoDrop 2000; Thermo Fisher Scientific Inc.). El cADN se sintetizó usando el kit QuantiTect $^{\circledR}$ Reverse Transcription (Qiagen, California, USA), siguiendo las instrucciones del fabricante, y fue amplificado mediante reacción en cadena de la polimerasa (PCR) empleando dNTPs (10 $\mathrm{mM}), \mathrm{MgCl}_{2}(25 \mathrm{mM})$, polimerasa Ecotaq y primers de los genes TNF- $\alpha$, factor nuclear kappa beta (NF$k \beta)$, CYP450, TGF- $\beta$, osteopontina (OPN), colágeno tipo IV alfa $V(\mathrm{COL} 4 \alpha 5)$ y factor de crecimiento hepático (HGF) (Tabla 1). Como normalizador se empleó el gen glucoronidasa beta (GUS $\beta$ ).

Los productos amplificados fueron sometidos a electroforesis en gel de agarosa, como fue descrito $^{13}$. Los niveles de expresión relativa de los genes fueron analizados por densitometría usando el software de análisis de imagen unidimensional QuantityOne ${ }^{\circledR}$ (BioRad, California, USA). 
Tabla 1. Características de los primers usados.

\begin{tabular}{|c|c|c|c|c|}
\hline Nombre (sigla) & $\begin{array}{l}\text { ID-Gen } \\
\text { Bank }\end{array}$ & Relación biológica & Secuencia & $\begin{array}{c}\text { Tamaño de } \\
\text { amplicon } \\
\text { (pb) }\end{array}$ \\
\hline $\begin{array}{l}\text { Glucoronidasa beta } \\
\text { (GUS } \beta \text { ) }\end{array}$ & 2990 & $\begin{array}{l}\text { Gen de referencia o normalizado. } \\
\text { Importante en la degradación } \\
\text { glicosaminoglicanos } \\
\text { proteoglicanos }\end{array}$ & $\begin{array}{l}\text { for: ATCACCGTCACCACCAGCGT } \\
\text { rev: } \\
\text { GTCCCATTCGCCACGACTTTGT }\end{array}$ & 131 \\
\hline $\begin{array}{l}\text { Factor de necrosis } \\
\text { tumoral alfa (TNF- } \alpha)\end{array}$ & 7124 & Inflamación y resistencia a insulina & $\begin{array}{l}\text { for: } \\
\text { GTGACAAGCCTGTAGCCCAT } \\
\text { rev: } \\
\text { CAGACTCGGCAAAGTCGAGA }\end{array}$ & 417 \\
\hline $\begin{array}{l}\text { Factor nuclear kappa } \\
\text { beta (NF-kß) }\end{array}$ & 222698 & $\begin{array}{l}\text { Regulador de la } \\
\text { inflamatoria }\end{array}$ & $\begin{array}{l}\text { for: CCCCGCAGAGCAGATGTT } \\
\text { rev: TCAGGCGCTCCCAATTCC }\end{array}$ & 319 \\
\hline $\begin{array}{l}\text { Citocromo P450 2E1 } \\
\text { (CYP2E1) }\end{array}$ & 1571 & Inducción de estrés oxidativo & $\begin{array}{l}\text { for: } \\
\text { TGCTGGAAGCACTCAGGAAG } \\
\text { rev: TCTCTGTCCCCGCAAAGAAC }\end{array}$ & 447 \\
\hline $\begin{array}{l}\text { Factor } \\
\text { crecimiento } \\
\text { transformante } \\
\text { (TGF- } \beta \text { B) }\end{array}$ & 655 & $\begin{array}{l}\text { Fibrogénesis, } \\
\text { daño a hepatocitos y producción } \\
\text { de citocinas inflamatorias }\end{array}$ & $\begin{array}{l}\text { for: } \\
\text { GGCAGCTGTACATTGACTTCC } \\
\text { rev: CCTTGCTGTACTGCGTGTCC }\end{array}$ & 129 \\
\hline Osteopontina (OPN) & 6696 & Fibrogénesis & $\begin{array}{l}\text { for: } \\
\text { GAATCTCCTAGCCCCACAGACC } \\
\text { rev: } \\
\text { CACGGCTGTCCCAATCAGAA }\end{array}$ & 464 \\
\hline $\begin{array}{l}\text { Colágeno tipo IV alfa } \\
\text { (V-COL4 } \alpha 5)\end{array}$ & 1288 & $\begin{array}{l}\text { Fibrogénesis y remodelación de } \\
\text { matriz extracelular }\end{array}$ & $\begin{array}{l}\text { for: AAATTCCCGGCTGGCTCTAA } \\
\text { rev: } \\
\text { TCCCCCAAATAACTGAGCGTC }\end{array}$ & 380 \\
\hline $\begin{array}{ll}\text { Factor } & \text { de } \\
\text { crecimiento } & \\
\text { hepático (HGF) } & \end{array}$ & 3082 & Regeneración hepática & $\begin{array}{l}\text { for: } \\
\text { CCTTCGAGCTATCGGGGTAAAG } \\
\text { rev: AGTGCCCCTGTAGCCTTCT }\end{array}$ & 459 \\
\hline
\end{tabular}

\section{Análisis estadístico}

Los datos fueron analizados utilizando el paquete estadístico IBM $^{\circledR}$ SPSS $^{\circledR}$ Statistic. El supuesto de normalidad fue evaluado a partir del test de ShapiroWilk y la homogeneidad de varianzas. Las variables que presentaron una distribución normal se describieron según su promedio y desviación estándar, mientras que aquellas que no se distribuían de forma normal, con la mediana y rango intercuartílico. Para comparar las diferencias de variables continuas entre los grupos, se utilizó tStudent o la prueba de Mann-Whitney según la distribución de los datos. Se aplicó la prueba de chi cuadrado para analizar las variables categóricas, y el valor de $p<0,05$ se consideró estadísticamente significativo. Las gráficas se realizaron en el programa Graphpad Prism ${ }^{\circledR}$.

\section{Declaración sobre aspectos éticos}

La investigación fue conducida de acuerdo con la Declaración de Helsinki de 1975 y la Resolución 8430 de 1993 del Ministerio de Salud de Colombia. La investigación fue aprobada por el Comité de Ética de la Universidad de Cartagena. Todos los participantes, previamente informados sobre el objetivo del estudio, firmaron consentimiento informado para que sus datos hicieran parte del estudio, así como para donar una muestra de sangre y otra de biopsia hepática para estudios moleculares. Los datos de los pacientes fueron tabulados de forma aleatoria y se anonimizaron totalmente para garantizar la protección de la confidencialidad. 


\section{RESULTADOS}

En el cribado clínico inicial se incluyeron 160 pacientes con enfermedad de hígado graso no alcohólico. De ellos, 104 (65\%) fueron descartados de la base de datos por los siguientes criterios: 18 debido a información incompleta, 23 por ausencia de interés para participar en el estudio, 37 por esteatohepatitis, 21 por cirrosis y cinco debido al uso de un fármaco hepatotóxico (isoniazida). Así, se incluyeron 56 pacientes en el estudio, todos ellos diagnosticados por criterios clínicos y ultrasonografía con esteatosis leve o grado 1 no alcohólica, sin presencia evidente de fibrosis: 38 mujeres $(67,9 \%)$ y 18 varones $(32,1 \%)$. El promedio de edad fue de 47,9 años $( \pm 14,5)$, con un intervalo de 20-92 años. Del total de los pacientes incluidos, 22 eran obesos, y 34 , no obesos.

En la tabla 2 se muestran las características sociodemográficas y antropométricas de los pacientes con esteatosis obesos y no obesos incluidos en este estudio. Como se puede observar, hubo predominio del género femenino en ambos grupos (obesos: 59,1\%; no obesos: 73,5\%). Además, la mayoría de los pacientes tanto obesos $(77,3 \%)$ como no obesos $(64,7 \%)$ provenían del área urbana (Tabla 2).

En relación con las diferentes variables bioquímicas estudiadas, se observaron diferencias estadísticamente significativas para ALT $(p=0,012)$, siendo mayor el valor de este analito en el grupo de no obesos (Tabla 3). Por su parte, el valor de la mediana de la ALP estuvo por encima de los límites de referencia (hombres <270 U/L; mujeres <240 $\mathrm{U} / \mathrm{L})$. Esta elevación se halló tanto en el grupo obesos (416 U/L) como en el de no obesos (361 U/L). Haciendo referencia a la población de estudio total, se encontró que el padecimiento considerado como componente del SM más frecuente fue la obesidad con $39,3 \%$, seguido de la HTA, presente en el 33,9\% de los pacientes, y la DM y dislipidemia con un $30,4 \%$ y $17,9 \%$ respectivamente. Al comparar el grupo de pacientes con esteatosis obesos y el grupo de pacientes con esteatosis no obesos con la presencia del $\mathrm{SM}$, los datos muestran diferencias significativas $(p=0,012)$. En el grupo de pacientes con esteatosis obesos, el $27,3 \%$ padecían de SMC, y el $72,7 \%$, de
$\mathrm{SMI}$, mientras que en los pacientes con esteatosis no obesos el $17,6 \%$ padecían de SMC, el 35,3\%, de SMI, y el $47,1 \%$ no padecía de ningún componente del SM (Tabla 3).

Se valoraron por RT-PCR los niveles de expresión de los genes TNF- $\alpha$, TGF- $\beta$, COL4 $\alpha 5$, HGF, OPN, CYP2E1 y NF-k $\beta$, relacionados con la inflamación, la remodelación de la matriz extracelular, fibrogénesis, inducción del estrés oxidativo y regeneración hepática. Los niveles de expresión de todos los genes evaluados fueron mayores en el grupo obesos que en el grupo de no obesos (Figura 1), con diferencias estadísticamente significativas para CYP2E1 ( $p=0,047$ ) (Figura 1c).

Al comparar los resultados de expresión génica con la presencia de $\mathrm{SM}$, se obtienen hallazgos interesantes: del $47,1 \%$ de los pacientes no obesos que no padecían de ningún componente del $\mathrm{SM}$, el $20,58 \%$ presentaban un IMC menor a $25 \mathrm{~kg} / \mathrm{m}^{2}$. En estos pacientes solo se observó la expresión del gen TGF- $\beta$. Por otra parte, la expresión de los genes OPN y HGF se detectó en ambos grupos, pero solo en individuos que presentaban HTA y DM (figuras 1e y $1 \mathrm{~g})$, lo cual corresponde al $32 \%$ en el grupo obesos y al $20 \%$ en el grupo de no obesos. 
Tabla 2. Características sociodemográficas y antropométricas de los pacientes con esteatosis no alcohólica.

\begin{tabular}{|c|c|c|c|c|c|c|c|}
\hline & \multicolumn{2}{|c|}{ Total pacientes $(\mathrm{N}=56)$} & \multicolumn{2}{|c|}{ Obesos $(\mathrm{N}=22)$} & \multicolumn{2}{|c|}{ No obesos $(\mathrm{N}=34)$} & $\mathbf{p}$ \\
\hline \multicolumn{8}{|c|}{ Variables sociodemográficas } \\
\hline Edad, $\overline{\mathbf{X}}( \pm)$ & 47,9 & $(14,5)$ & 44,2 & $(12,2)$ & 50,1 & $(15,3)$ & 0,135 \\
\hline \multicolumn{8}{|l|}{ Género, n (\%) } \\
\hline Femenino & 38 & $(67,9)$ & 13 & $(59,1)$ & 25 & $(73,5)$ & \multirow[t]{2}{*}{0,380} \\
\hline Masculino & 18 & $(32,1)$ & 9 & $(40,9)$ & 9 & $(26,5)$ & \\
\hline \multicolumn{8}{|c|}{ Procedencia, n (\%) } \\
\hline Urbano & 39 & $(69,6)$ & 17 & $(77,3)$ & 22 & $(64,7)$ & \multirow[t]{2}{*}{0,549} \\
\hline Rural & 17 & $(30,4)$ & 5 & $(22,7)$ & 12 & $(35,3)$ & \\
\hline \multicolumn{8}{|c|}{ Régimen de afiliación, n (\%) } \\
\hline Contributivo & 10 & $(17,9)$ & 4 & $(18,2)$ & 6 & $(17,6)$ & \multirow[t]{2}{*}{1,000} \\
\hline Subsidiado & 47 & $(82,1)$ & 18 & $(81,8)$ & 28 & $(82,4)$ & \\
\hline \multicolumn{8}{|c|}{ Medidas antropométricas } \\
\hline Peso, Me (RI) & 75,5 & $(67,2-91,7)$ & 94,5 & $(84,5-103)$ & 68,7 & $(65-75,2)$ & $0,000^{*}$ \\
\hline Talla, $\bar{x}( \pm)$ & 1,7 & $(0,1)$ & 1,6 & $(0,09)$ & 1,65 & $(0,08)$ & 0,310 \\
\hline $\mathrm{IMC}, \mathrm{Me}(\mathrm{RI})$ & 28 & $(24,8-31,4)$ & 33,2 & $(30,8-35,7)$ & 26,8 & $(24,0-27,8)$ & $0,000^{*}$ \\
\hline
\end{tabular}

X: media; \pm : desviación estándar; Me: mediana. La edad fue medida en años; el peso, en kilogramos, y la talla, en metros.

Tabla 3. Características clínicas de los pacientes con esteatosis no alcohólica.

\begin{tabular}{|c|c|c|c|c|c|c|c|}
\hline & \multicolumn{2}{|c|}{ Total $(\mathrm{N}=56)$} & \multicolumn{2}{|c|}{ Obesos ( $\mathrm{N}=22$ ) } & \multicolumn{2}{|c|}{ No obesos ( $\mathrm{N}=34)$} & $p$ \\
\hline \multicolumn{8}{|c|}{ Mediciones bioquímicas, Me (RI) } \\
\hline $\mathrm{ALT}(\mathrm{U} / \mathrm{L})$ & 35 & $(19,2-100,5)$ & 26,5 & $(14,7-45,2)$ & 68 & $(24,7-178,7)$ & $0,012^{*}$ \\
\hline AST (U/L) & 35,5 & $(19-76,2)$ & 25,5 & $(19-48,7)$ & 56 & $(21,5-97)$ & 0,052 \\
\hline AST/ALT & 0,9 & $(0,94-1,01)$ & 1,16 & $(0,67-1,87)$ & 0,84 & $(0,55-1,34)$ & 0,123 \\
\hline Bilirrubina total (mg/dL) & 0,6 & $(0,39-0,88)$ & 0,72 & $(0,42-0,85)$ & 0,55 & $(0,33-1,30)$ & 0,731 \\
\hline Bilirrubina directa $(\mathrm{mg} / \mathrm{dL})$ & 0,31 & $(0,2-0,58)$ & 0,34 & $(0,19-0,49)$ & 0,3 & $(0,20-1,01)$ & 0,755 \\
\hline Bilirrubina indirecta $(\mathrm{mg} / \mathrm{dL})$ & 0,2 & $(0,12-0,35)$ & 0,2 & $(0,12-0,41)$ & 0,2 & $(0,11-0,31)$ & 0,820 \\
\hline Albumina $(\mathrm{g} / \mathrm{L})$ & 39,9 & $(37,9-42,8)$ & 39 & $(38-40,92)$ & 40,4 & $(37,3-43,7)$ & 0,481 \\
\hline Glicemia (mg/dL) & 109,6 & $(91,7-141,3)$ & 105,3 & $(85,1-117,3)$ & 113,9 & $(95,2-146,2)$ & 0,196 \\
\hline Colesterol total (mg/dL) & 169,4 & $(150,4-188,1)$ & 164,3 & $147,2-185,7)$ & 170,1 & $(151,8-189,8)$ & 0,557 \\
\hline Triglicéridos (mg/dL) & 149,4 & $(134,2-221,6)$ & 148,09 & $(130,4-224,1$ & 149,6 & $(136,4-222,2)$ & 0,750 \\
\hline $\operatorname{ALP}(U / L)$ & 361 & $(316-416)$ & 416 & $(277-416)$ & 361 & $(318-402)$ & 0,423 \\
\hline Creatinina $(\mathrm{mg} / \mathrm{dL})$ & 0,94 & $(0,76-1,16)$ & 1 & $(0,8-1,5)$ & 0,83 & $(0,71-1,00)$ & $0,002 *$ \\
\hline \multicolumn{8}{|l|}{ Síndrome metabólico, n (\%) } \\
\hline Completo & 12 & $(21,4)$ & 6 & $(27,3)$ & 6 & $(17,6)$ & \multirow{3}{*}{$0,012 *$} \\
\hline Incompleto & 28 & (50) & 16 & $(72,7)$ & 12 & $(35,3)$ & \\
\hline Sin síndrome metabólico & 16 & $(28,6)$ & 0 & $(0,0)$ & 16 & $(47,1)$ & \\
\hline
\end{tabular}

Me: mediana; RI: rango intercuartil; ALT: alanino aminotransferasa; AST: aspartato aminotransferasa; TP: tiempo de protrombina; TPT: tiempo parcial de tromboplastina; ALP: fosfatasa alcalina international normalized ratio.

*El nivel de significancia es 0,05 . 

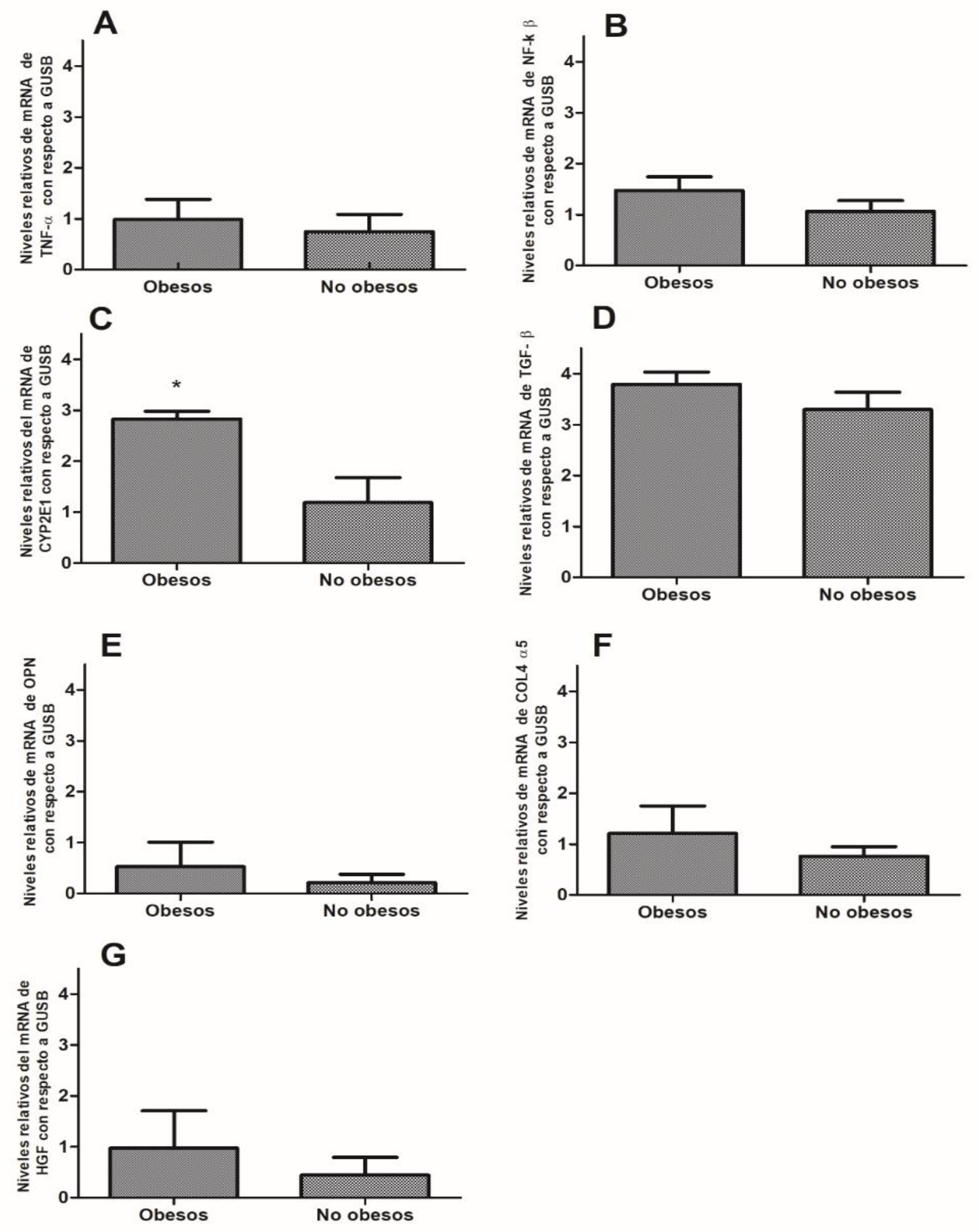

Figura 1. Expresión génica de los genes evaluados en biopsias de tejido hepático de individuos con esteatosis no alcohólica: a) niveles relativos de expresión de mRNA de TNF- $\alpha$ en individuos obesos y no obesos con esteatosis no alcohólica; b) niveles relativos de expresión de mRNA de NF-k $\beta$ en individuos obesos y no obesos con esteatosis no alcohólica; $c$ ) niveles relativos de expresión de mRNA de CYP2E1 en individuos obesos y no obesos con esteatosis no alcohólica; $d$ ) niveles relativos de expresión de mRNA de TGF- $\beta$ en individuos obesos y no obesos con esteatosis no alcohólica; e) niveles relativos de expresión de mRNA de OPN en individuos obesos y no obesos con esteatosis no alcohólica; f) niveles relativos de expresión de mRNA de COL4 $\alpha 5$ en individuos obesos y no obesos con esteatosis no alcohólica; g) niveles relativos de expresión de mRNA de HGF en individuos obesos y no obesos con esteatosis no alcohólica. 
Se valoraron por RT-PCR los niveles de expresión de los genes TNF- $\alpha$, TGF- $\beta$, COL4 $\alpha 5$, HGF, OPN, CYP2E1 y NF-k $\beta$, relacionados con la inflamación, la remodelación de la matriz extracelular, fibrogénesis, inducción del estrés oxidativo y regeneración hepática. Los niveles de expresión de todos los genes evaluados fueron mayores en el grupo obesos que en el grupo de no obesos (Figura 1), con diferencias estadísticamente significativas para CYP2E1 ( $p=0,047)$ (Figura 1c).

Al comparar los resultados de expresión génica con la presencia de $\mathrm{SM}$, se obtienen hallazgos interesantes: del $47,1 \%$ de los pacientes no obesos que no padecían de ningún componente del SM, el $20,58 \%$ presentaban un IMC menor a $25 \mathrm{~kg} / \mathrm{m}^{2}$. En estos pacientes solo se observó la expresión del gen TGF- $\beta$. Por otra parte, la expresión de los genes OPN y HGF se detectó en ambos grupos, pero solo en individuos que presentaban HTA y DM (figuras 1e y $1 \mathrm{~g})$, lo cual corresponde al $32 \%$ en el grupo obesos y al $20 \%$ en el grupo de no obesos.

\section{DISCUSIÓN}

Si bien la esteatosis se asocia con pronóstico benigno, estudios actuales sugieren que la progresión a fibrosis en estos casos es posible ${ }^{3-5}$. Actualmente, no existe una forma definitiva para conocer qué pacientes y bajo qué factores tienen mayor riesgo de progresar a esteatohepatitis $y$ fibrosis con miras a brindar una intervención temprana. Además, los estudios de NAFLD en personas no obesas y sobre los factores que contribuyen a su progresión en la etapa inicial son escasos.

Este estudio en pacientes con esteatosis obesos y no obesos muestra datos interesantes que incluyen la posible activación precoz de vías de señalización que conducen a fibrosis en pacientes con esteatosis leve, ante la presencia de componentes del SM. En esta investigación, el $67,9 \%$ de los pacientes pertenecía al género femenino, resultados concordantes con otros estudios en locales ${ }^{12,14}$. Este mayor porcentaje de mujeres con NALFD sugiere que las hormonas sexuales podrían desempeñar un papel relevante en el desarrollo de esteatosis ${ }^{15}$, tal como se reporta en un estudio in vivo que demuestra que los andrógenos pueden proteger contra NALFD ${ }^{16}$. No obstante, los reportes de otros autores han documentado mayor frecuencia de NAFLD en hombres ${ }^{17,18}$. En este sentido es importante considerar que la menor frecuencia del género masculino aquí reportada posiblemente se debe al criterio de exclusión de consumo de alcohol de este trabajo, teniendo en cuenta que en la costa Caribe dicho consumo es mayor en hombres, en especial en estratos socioeconómicos bajos ${ }^{19,20}$. Además, se podría inferir que la mayoría de la población de estudio correspondía precisamente a ese nivel económico, pues el $82,1 \%$ pertenecían al régimen de salud subsidiado.

Con referencia a la procedencia, se confirma lo reportado en la literatura ${ }^{21}$, que apunta a mayor frecuencia de NAFLD en área urbana. Esta tendencia se ve soportada por los procesos rápidos de urbanización que dirigen a estilos de vida con reducida actividad física y malos hábitos alimenticios que incluyen mayor consumo de alimentos procesados con alto contenido de grasas y calorías, lo cual incrementa la incidencia de comorbilidades asociadas con NAFLD ${ }^{22}$.

En lo concerniente a las variables bioquímicas, el incremento de la ALP observado en ambos grupos también ha sido descrito por Pantsari et al., quienes sugieren que las mujeres adultas con ALP elevada y factores de riesgo para NAFLD tienen una mayor probabilidad de presentar esteatohepatitis $y$ fibrosis $^{23}$. Los estudios previos también han encontrado diferencias en la ALT de pacientes con esteatosis obesos y no obesos ${ }^{24,25}$, lo cual coincide con los hallazgos de esta investigación de una ALT aumentada en el grupo de pacientes con esteatosis no obesos, posiblemente indicando daño hepático por acumulación de lípidos en este tipo de población y el progreso de la enfermedad.

Adicionalmente, los resultados evidenciaron que más del $70 \%$ de la población estudiada padecía de por lo menos un componente del SM; cifras alarmantes teniendo en cuenta que la presencia de un componente anticipa la aparición de los demás ${ }^{26}$. Tanto NAFLD como el SM comparten muchas anomalías en el metabolismo de la glucosa y lípidos, y por esa razón los individuos con NAFLD 
generalmente tienen SM o uno de sus componentes $^{27}$, como se evidencia en los resultados.

Los niveles de expresión génica hallados en el presente estudio son consistentes con diferentes estudios que sugieren que en hígado esteatósico con componentes del SM la acumulación de ácidos grasos estimula la producción de citoquinas proinflamatorias, como TNF- $\alpha$, que induce la inflamación hepática por la activación de la vía NF$k \beta^{28,29}$. También se encuentran concordancias con estudios realizados en modelos animales que demuestran la activación de la vía de señalización TGF- $\beta$ en el progreso de la esteatosis y la participación de TGF- $\beta$ en el inicio de la acumulación de COL4a5. Esto conduce a fibrogénesis en etapas tempranas de NAFLD y, en condiciones adicionales de componentes del SM, tiende a ser mayor la activación de estas vías de señalización ${ }^{30}$, tal como se sugiere en esta investigación por la expresión tanto de TGF- $\beta$ como de COL $4 \alpha 5$ en pacientes con esteatosis obesos y no obesos.

Es importante destacar que $20,58 \%$ de los pacientes con esteatosis del grupo no obesos no padecían de ningún componente del SM y presentaron un IMC menor a $25 \mathrm{~kg} / \mathrm{m}^{2}$. En estos individuos solo se observó la expresión del gen TGF- $\beta$, lo cual apoya el principio de la contribución del SM en la patogenia de NAFLD y sugiere que, de los genes evaluados, el gen TGF- $\beta$ se activa tempranamente como participante de las vías que conducen a fibrogénesis en hígado esteatósico de individuos no obesos sin componente del SM.

El mayor nivel de expresión de CYP2E1 estadísticamente significativo en obesos podría indicar una señal de alarma por un posible progreso de la esteatosis, debido a que la sobrecarga de ácidos grasos per se también puede inducir a mayor activación del gen CYP2E1 y, por consiguiente, a mayor producción de especies reactivas de oxígeno (ROS) en la mitocondria, promoviendo la activación de NF-k $\beta$. Además, CYP2E1 participa en la regulación lipídica, y su sobreexpresión puede promover carcinogénesis hepática ${ }^{30,31}$.

Finalmente, la expresión de OPN y HFG observada podría deberse a la existencia de una lesión hepática mayor ocasionada no solo por la esteatosis, sino también por la presencia de componentes del SM. Considerando que el gen OPN está asociado con la transición de células estrelladas en miofibroblastos productores de colágeno ${ }^{32}$ y el gen HFG se relaciona con la regeneración crónica del hígado a partir de la activación de células madres ${ }^{33}$, los datos obtenidos podrían sugerir que la OPN puede activarse específicamente en la inducción de células progenitoras juntamente con la vía de señalización HGF para contribuir a la reparación del hígado lesionado. De manera que, a futuro, se sugiere realizar estudios que incluyan otros genes asociados a vías de HGF/cMet y OPN que participan en la transición de células estrelladas.

Asimismo, los resultados sugieren que, de los genes estudiados en hígado esteatósico de pacientes no obesos, el gen TGF- $\beta$ se activa tempranamente como participante de las vías que conducen a fibrogénesis, y en hígado esteatósico de pacientes obesos con SMC el nivel de expresión significativo de CYP2E1 podría indicar una señal de alarma por un posible progreso de la enfermedad. Debe prestarse especial atención y seguimiento de la esteatosis no alcohólica en pacientes obesos con otros componentes del síndrome metabólico.

\section{AGRADECIMIENTOS}

Agradecimientos a las entidades financiadoras de este proyecto: Colciencias por su apoyo con la financiación a través del programa Jóvenes Investigadores e Innovadores, Convocatoria 7612016, y a la Universidad de Cartagena, Colombia, por el programa de fortalecimiento del grupo Prometeus (acta 036). Así también agradecimientos por el apoyo y colaboración en la ejecución de la investigación al Hospital Universitario del Caribe.

\section{DECLARACIÓN SOBRE CONFLICTOS DE INTERESES}

Los autores declaran no tener conflicto de interés, que son independientes con respecto a las instituciones financiadoras y de apoyo, y que durante el proceso de ejecución del trabajo o redacción del manuscrito no han incidido intereses distintos a los que usualmente tiene la investigación. 


\section{CONTRIBUCIÓN DE LOS AUTORES}

Primer autor: diseño, metodología, recolección de información, análisis estadístico, redacción y revisión.

Segundo autor: metodología, recolección de información, análisis estadístico y revisión.

Tercer autor: recolección de información, escritura y revisión.

Cuarto autor: diseño, metodología y revisión.

Quinto autor: diseño, escritura y revisión.

\section{REFERENCIAS BIBLIOGRÁFICAS}

1.Fazel $Y$, Koenig $A B$, Sayiner $M$, Goodman $Z D$, Younossi ZM. Epidemiology and natural history of non-alcoholic fatty liver disease. Metabolism. 2016; 65(8): $\quad$ 1017-25. Doi: http://dx.doi.org/10.1016/j.metabol.2016.01.012.

2.Ipsen DH, Lykkesfeldt J, Tveden-Nyborg P. Molecular mechanisms of hepatic lipid accumulation in non-alcoholic fatty liver disease. Cell Mol Life Sci. 2018; 75(18): 3313-27. Doi: http://dx.doi.org/10.1007/s00018-018-2860-6.

3.McPherson S, Hardy T, Henderson E, Burt AD, Day $\mathrm{CP}$, Anstee QM. Evidence of NAFLD progression from steatosis to fibrosing-steatohepatitis using paired biopsies: Implications for prognosis and clinical management. J Hepatol. 2015; 62(5): 1148-55. Doi: http://dx.doi.org/10.1016/j.jhep.2014.11.034.

4.Wong VW, Wong GL, Choi PC, Chan AW, Li MK, Chan HY, et al. Disease progression of non-alcoholic fatty liver disease: a prospective study with paired liver biopsies at 3 years. Gut. 2010; 59(7): 969-74. Doi: http://dx.doi.org/10.1136/gut.2009.205088.

5.Pais R, Charlotte F, Fedchuk L, Bedossa P, Lebray $P$, Poynard $T$, et al. A systematic review of follow-up biopsies reveals disease progression in patients with non-alcoholic fatty liver. J Hepatol. 2013; 59(3): 5506.

Doi: http://dx.doi.org/10.1016/j.jhep.2013.04.027.

6.Di Costanzo A, D'Erasmo L, Polimeni L, Baratta F, Coletta P, Di Martino M, et al. Non-alcoholic fatty liver disease and subclinical atherosclerosis: A comparison of metabolically- versus geneticallydriven excess fat hepatic storage. Atherosclerosis. 2017; 257: 232-9. Doi: http://dx.doi.org/10.1016/j.atherosclerosis.2016.1 2.018 .

7.Cayon A, Crespo J, Guerra AR, Pons-Romero F. Gene expression in obese patients with nonalcoholic steatohepatitis. Rev Esp Enferm Dig. 2008; 100(4): 212-8. Doi: http://dx.doi.org/10.4321/s113001082008000400004.

8. Walton KL, Johnson KE, Harrison CA. Targeting TGF- $\beta$ Mediated SMAD Signaling for the Prevention of Fibrosis. Front Pharmacol. 2017; 8: 461. Doi: http://dx.doi.org/10.3389/fphar.2017.00461.

9.Aljomah G, Baker S, Liu W, Kozielski R, Oluwole J, Lupu B, et al. Induction of CYP2E1 in non-alcoholic fatty liver diseases. Exp Mol Pathol. 2015; 99(3): 677-81.

Doi: http://dx.doi.org/10.1016/j.yexmp.2015.11.008.

10.Sanyal AJ. AGA technical review on nonalcoholic fatty liver disease. Gastroenterology. 2002; 123(5): 1705-25. Doi: http://dx.doi.org/10.1053/gast.2002.36572.

11.Mustapic S, Ziga S, Matic V, Bokun T, Radic B, Lucijanic $M$, et al. Ultrasound Grade of Liver Steatosis Is Independently Associated with the Risk of Metabolic Syndrome. Can J Gastroenterol Hepatol. 2018; 2018: 8490242. Doi: http://dx.doi.org/10.1155/2018/8490242.

12.Lambis L, Solana J, Gastelbondo B, Romero D, Garrido D, Puello $W$, et al. Factores de riesgo asociados a hígado graso de origen no alcohólico en una población del Caribe Colombiano. Rev Colomb de Gastroenterol. 2016; 31(2): 89-95. Doi: http://dx.doi.org/10.22516/25007440.77.

13.Madera M, González F, Romero D, Suárez A. Expresión Génica del Factor de Crecimiento Transformante Beta en Niños con Fisura Labio Palatina no Sindrómica. Int J Odontostomat. 2016; 10(1): 75-84. Doi: http://dx.doi.org/10.4067/S0718$381 \times 2016000100013$. 
14.Ochoa CE, Calambás F. Hígado graso no alcohólico en consulta de gastroenterología. Repert Med Cir. 2017; 26(4): 225-30. Doi: http://dx.doi.org/10.1016/j.reper.2017.10.005.

15.Kur P, Kolasa-Wołosiuk A, Misiakiewicz-Has K, Wiszniewska B. Sex Hormone-Dependent Physiology and Diseases of Liver. Int J Environ Res Public Health. 2020; 17(8): 2620. Doi: http://dx.doi.org/10.3390/ijerph17082620.

16.Zhang $\mathrm{H}$, Liu Y, Wang L, Li Z, Zhang $\mathrm{H}$, Wu J, et al. Differential effects of estrogen/androgen on the prevention of nonalcoholic fatty liver disease in the male rat. J Lipid Res. 2013; 54(2): 345-57. Doi: http://dx.doi.org/10.1194/jlr.M028969.

17.Sweet P, Khoo T, Nguyen S. Nonalcoholic Fatty Liver Disease. Prim Care. 2017; 44(4): 599-607. Doi: http://dx.doi.org/10.1016/j.pop.2017.07.003.

18. Katikireddi SV, Whitley E, Lewsey J, Gray L, Leyland AH. Socioeconomic status as an effect modifier of alcohol consumption and harm: analysis of linked cohort data. Lancet Public Health. 2017; 2(6): $\quad$ e267-e76. Doi: http://dx.doi.org/10.1016/s2468-2667(17)30078-6.

19.De la Espriella Guerrero RA, Rodriguez V, Rincón CJ, Morales DC, Rodríguez SJ, Gómez-Restrepo C. Consumo de alcohol en la población colombiana. Encuesta Nacional de Salud Mental 2015. Rev Colomb Psiquiatr. 2016; 45(1): 76-88. Doi: http://dx.doi.org/10.1016/j.rcp.2016.05.002.

20.Pérez-Gómez A, Lanziano C, Reyes-Rodríguez MF, Mejía-Trujillo J, Cardozo-Macías F. Perfiles asociados al consumo de alcohol en adolescentes colombianos. Acta Colomb de Psicol. 2018; 21(2): 258-69.

Doi: http://dx.doi.org/10.14718/ACP.2018.21.2.12.

21.Lee K, Sung J, Kim J, Park T. The roles of obesity and gender on the relationship between metabolic risk factors and non-alcoholic fatty liver disease in Koreans. Diabetes Metab Res Rev. 2009; 25(2): 1505. Doi: http://dx.doi.org/10.1002/dmrr.924.
22. Hallsworth K, Adams LA. Lifestyle modification in NAFLD/NASH: Facts and figures. JHEP Rep. 2019; 1(6): 468-79. Doi: http://dx.doi.org/10.1016/j.jhepr.2019.10.008.

23.Pantsari MW, Harrison SA. Nonalcoholic Fatty Liver Disease Presenting With an Isolated Elevated Alkaline Phosphatase. J Clin Gastroenterol. 2006; 40(7): 633-5. Doi: http://dx.doi.org/10.1097/00004836-20060800000015.

24.Vendhan R, Amutha A, Anjana RM, Unnikrishnan R, Deepa M, Mohan V. Comparison of Characteristics Between Nonobese and Overweight/Obese Subjects with Nonalcoholic Fatty Liver Disease in a South Indian population. Diabetes Technol Ther. 2014; 16(1): 48-55. Doi: http://dx.doi.org/10.1089/dia.2013.0165.

25.Kwak J, Jun D, Lee S, Cho Y, Lee K, Lee H, et al. Lifestyle predictors of obese and non-obese patients with nonalcoholic fatty liver disease: A crosssectional study. Clin Nutr. 2018; 37(5): 1550-7. Doi: http://dx.doi.org/10.1016/j.clnu.2017.08.018.

26. Hennig EE, Mikula M, Goryca K, Paziewska A, Ledwon J, Nesteruk $M$, et al. Extracellular matrix and cytochrome P450 gene expression can distinguish steatohepatitis from steatosis in mice. J Cell Mol Med. 2014; 18(9): 1762-72. Doi: http://dx.doi.org/10.1111/jcmm.12328.

27.Kwon OW, Jun DW, Lee SM, Lee KN, Lee HL, Lee $\mathrm{OY}$, et al. Carbohydrate but not fat is associated with elevated aminotransferases. Aliment Pharmacol Ther. 2012; 35(9): 1064-72. Doi: http://dx.doi.org/10.1111/j.1365-

2036.2012.05061.x.

28.Seo YY, Cho YK, Bae J-C, Seo MH, Park SE, Rhee E$J$, et al. Tumor Necrosis Factor- $\alpha$ as a Predictor for the Development of Nonalcoholic Fatty Liver Disease: A 4-Year Follow-Up Study. Endocrinol Metab (Seoul). 2013; 28(1): 41-5. Doi: http://dx.doi.org/10.3803/EnM.2013.28.1.41.

29.Tan Y, Kim J, Cheng J, Ong M, Lao W-G, Jin X-L, et al. Green tea polyphenols ameliorate non-alcoholic fatty liver disease through upregulating AMPK 
activation in high fat fed Zucker fatty rats. World J Gastroenterol. 2017; 23(21): 3805-14. Doi: http://dx.doi.org/10.3748/wjg.v23.i21.3805.

30.Yang L, Roh YS, Song J, Zhang B, Liu C, Loomba R, et al. Transforming growth factor beta signaling in hepatocytes participates in steatohepatitis through regulation of cell death and lipid metabolism in mice. Hepatology. 2014; 59(2): 483-95. Doi: http://dx.doi.org/10.1002/hep.26698.

31.De Lédinghen $V$, Liu $H$, Zhang $F$, Lo $C R$, Subbaramaiah K, Dannenberg AJ, et al. Induction of cyclooxygenase-2 by tumor promoters in transformed and cytochrome P450 2E1-expressing hepatocytes. Carcinogenesis. 2002; 23(1): 73-9. Doi: http://dx.doi.org/10.1093/carcin/23.1.73.

32.Wen $Y$, Jeong $S$, Xia $Q$, Kong $X$. Role of Osteopontin in Liver Diseases. Int J Biol Sci. 2016; 12(9): $\quad$ 1121-8. Doi: http://dx.doi.org/10.7150/ijbs.16445.

33.Suárez-Causado A, Caballero-Díaz D, Bertrán E, Roncero C, Addante A, García-Álvaro $M$, et al. $\mathrm{HGF} / \mathrm{c}-$ Met signaling promotes liver progenitor cell migration and invasion by an epithelialmesenchymal transition-independent, phosphatidyl inositol-3 kinase-dependent pathway in an in vitro model. Biochim Biophys Acta. 2015; 1853(10 Pt A): 2453-63. Doi:

http://dx.doi.org/10.1016/j.bbamcr.2015.05.017 